VoL. 47 (1993) [473-481]

\title{
ON RELATIONS BETWEEN JACOBIANS AND RESULTANTS OF POLYNOMIALS IN TWO VARIABLES
}

\author{
TAKIS SaKKalis
}

This paper investigates some of the connections between the zeros of a polynomial vector field $F=(f, g): \mathbb{C}^{2} \rightarrow \mathbb{C}^{2}$ and the Jacobian determinant $J(f, g)$ of $f$ and $g$. As a consequence, sufficient conditions are given for $F$ to have no zeros. In addition, in the case where $F$ has an inverse $F^{-1}$, it is proven that $F^{-1}$ is also polynomial.

\section{INTRODUCTION}

Let $f(x, y), g(x, y)$ be nonzero polynomials with coefficients in $\mathbb{C}$, and let $F=$ $(f, g): \mathbb{C}^{2} \rightarrow \mathbb{C}^{2}$. A zero of $F$ is a point $\left(x_{0}, y_{0}\right) \in \mathbb{C}^{2}$ with the property $F\left(x_{0}, y_{0}\right)=$ $(0,0)$.

In this paper we investigate some of the connections between zeros of $F$ and the Jacobian determinant $J(f, g)$ of $f$ and $g$. This leads to the consideration of resultants of the type $\operatorname{Res}_{y}(f-u, g-v)=A(x, u, v), \operatorname{Res}_{x}(f-u, g-v)=B(y, u, v)$, where $u$ and $v$ are indeterminates. Let $k, r$ be the degrees of $A(x, u, v)$ in $x$ and $B(y, u, v)$ in $y$, respectively. Theorem 1 of Section 3 gives necessary and sufficient conditions for $k$ and $r$ to be zero in terms of $J(f, g)$. As a consequence, sufficient conditions are given for $F$ to have no zeros.

In the case where $F$ is $1-1$ and onto, we show (Section 4) that $k=r=1$. Furthermore, $A(x, u, v)=a x+A_{0}(u, v), B(y, u, v)=b y+B_{0}(u, v)$, (Lemma 2), and this gives rise to the well-known fact that $F$ has a polynomial inverse, $F^{-1}$; Proposition 1 specifically computes $F^{-1}$. The McKay-Wang inversion formula which generalises Cramer's rule to two polynomials in two variables, was first derived in [3] and rederived by Adjamagbo and van den Essen in [1]. Our Proposition 1 also rederives this formula by using a different approach. As a result, $F$ is completely determined by its "border polynomials". We conclude with a conjecture regarding the nonexistence of zeros of $F$.

Received 15th June 1992

Copyright Clearance Centre, Inc. Serial-fee code: $0004-9729 / 93 \quad \$ A 2.00+0.00$. 


\section{Properties of Resultants}

Throughout this paper $f(x, y)$ and $g(x, y)$ are polynomials in $x, y$ with coefficients in the field $\mathbb{C}$ of complex numbers. We begin with the following definitions: The Jacobian determinant, $J(f, g)$, of $f$ and $g$ is defined by

$$
J(f, g)=\left|\begin{array}{ll}
\frac{\partial f}{\partial x} & \frac{\partial f}{\partial y} \\
\frac{\partial g}{\partial x} & \frac{\partial g}{\partial y}
\end{array}\right| .
$$

Let

$$
\begin{aligned}
& a(t)=a_{n} t^{n}+\cdots+a_{1} t+a_{0} \\
& b(t)=b_{m} t^{m}+\cdots+b_{1} t+b_{0}
\end{aligned}
$$

be nonzero polynomials of degrees $n$ and $m$ respectively, with coefficients in an integral domain $D$. The resultant of $a, b$ with respect to $t, \operatorname{Res}_{t}(a, b)$, is the following $(m+n) \times(m+n)$ determinant:

$$
\operatorname{Res}_{t}(a, b)=\left|\begin{array}{ccccccc}
a_{n} & a_{n-1} & \ldots & a_{0} & & & \\
& a_{n} & \ldots & a_{1} & a_{0} & & \\
& & \ddots & & & \ddots & \\
& & & a_{n} & \ldots & \ldots & a_{0} \\
b_{m} & \ldots & \ldots & \ldots & b_{0} & & \\
& \ddots & & & & \ddots & \\
& & b_{m} & \ldots & \ldots & \ldots & b_{0}
\end{array}\right|
$$

There are several well-known properties of resultants, $[2,6]$. We state here those that will be needed later.

Property 1. There are polynomials $A(t), B(t) \in D[t]$ of degrees $n^{\prime}, m^{\prime}$ respectively, $n^{\prime}<m, m^{\prime}<n$ so that

$$
a(t) A(t)+b(t) B(t)=\operatorname{Res}_{t}(a, b)
$$

Property 2. $\operatorname{Res}_{t}(a, b)=0 \Leftrightarrow a(t)$ and $b(t)$ have a common factor of positive degree. PROPERTY 3. Let $a(x, y)=a_{n} x^{n}+a_{n-1}(y) x^{n-1}+\cdots+a_{1}(y) x+a_{0}(y), b(x, y)=$ $b_{m} x^{m}+b_{m-1}(y) x^{m-1}+\cdots+b_{1}(y) x+b_{0}(y) \in \mathbb{C}[y][x]$, with $a_{n}, b_{m}$ nonzero complex numbers, and consider $p(y)=\operatorname{Res}_{x}(a, b)$. If $y_{0} \in \mathbb{C}$ is a root of $p(y)$, then there exists an $x_{0} \in \mathbb{C}$ with the property $a\left(x_{0}, y_{0}\right)=b\left(x_{0}, y_{0}\right)=0$. 
Property 4. Let $a(t)=a_{n} \prod_{i=1}^{n}\left(t-\alpha_{i}\right), b(t)=b_{m} \prod_{j=1}^{m}\left(t-\beta_{j}\right)$ be the factorisations of $a(t), b(t)$ in some splitting field $E$ of $a, b$ over the quotient field of $D$. Then

$$
\operatorname{Res}_{t}(a, b)=a_{n}^{m} b_{m}^{n} \prod_{i=1}^{n} \prod_{j=1}^{m}\left(\alpha_{i}-\beta_{j}\right)=a_{n}^{m} \prod_{i=1}^{n} b\left(\alpha_{i}\right)=(-1)^{m n} b_{m}^{n} \prod_{j=1}^{m} a\left(\beta_{j}\right)
$$

PROPERTY 5. $\operatorname{Res}_{t}(a, b c)=\operatorname{Res}_{t}(a, b) \operatorname{Res}_{t}(a, c)$, for any nonzero $c \in D[t]$.

DEFINITION 1. Let $p(x, y)$ be a polynomial with coefficients in $D$ whose.degree in $x$ is $n$. We say that $p$ is quasi-regular in $x$ if the coefficient of $x^{n}$ in $p(x, y)$ is a nonzero constant.

Let us now consider polynomials $f(x, y), g(x, y)$ so that their degress in $x$ and in $y$ are positive. Since we are going to consider resultants of $f$ and $g$ with respect to $x$ and $y$, in view of Property 3 , we shall henceforth assume, unless otherwise stated, that $f$ and $g$ are quasi-regular in both $x$ and $y$.

\section{A First Relation}

Let $u, v$ be indeterminates. Consider

$$
\begin{aligned}
& A(x, u, v)=\operatorname{Res}_{y}(f-u, g-v) \\
& B(y, u, v)=\operatorname{Res}_{x}(f-u, g-v)
\end{aligned}
$$

and write

$$
\begin{aligned}
& A(x, u, v)=A_{k}(u, v) x^{k}+\cdots+A_{1}(u, v) x+A_{0}(u, v) \\
& B(y, u, v)=B_{r}(u, v) y^{r}+\cdots+B_{1}(u, v) y+B_{0}(u, v)
\end{aligned}
$$

Our aim is to investigate the connection between the degrees $k$ and $r$ of $A, B$ and the nature of the polynomials $f$ and $g$.

The following theorem provides a necessary and sufficient condition for $k$ and $r$ to be zero.

THEOREM 1. Let $f(x, y)$ and $g(x, y)$ be quasi-regular in $x$ as well as in $y$. Let $A(x, u, v), B(y, u, v), k, r$ be as above. Then, the following conditions are equivalent:
(i) $k=0$.
(ii) $r=0$.
(iii) $\exists \varphi(u, v), \varphi \neq 0$, with $\varphi(f, g)=0$.
(iv) $J(f, g)=0$. 
Proof: We first note that $A_{0}(u, v) B_{0}(u, v) \neq 0$ : Since $f$ and $g$ are quasi-regular in $y$, it follows that

$$
\left.\operatorname{Res}_{y}(f-u, g-v)\right|_{x=0}=\operatorname{Res}_{y}(f(0, y)-u, g(0, y)-v) \neq 0,
$$

hence $A_{0}(u, v)=A(0, u, v) \neq 0$. Similarly, by the regularity in $x, B_{0}(u, v) \neq 0$.

(i) $\Rightarrow$ (ii). We argue by contradiction. Suppose then that $r \geqslant 1$. In that case pick $\left(u_{0}, v_{0}\right) \in \mathbb{C}^{2}$ so that $B_{r}\left(u_{0}, v_{0}\right) A_{0}\left(u_{0}, v_{0}\right) \neq 0$, and let $y_{0} \in \mathbb{C}$ be such that $B\left(y_{0}, u_{0}, v_{0}\right)=0$. By Property 3 , we can find $x_{0} \in \mathbb{C}$ with the property that $f\left(x_{0}, y_{0}\right)-$ $u_{0}=g\left(x_{0}, y_{0}\right)-v_{0}=0$. Then

$0=\operatorname{Res}_{y}\left(f\left(x_{0}, y\right)-u_{0}, g\left(x_{0}, y\right)-v_{0}\right)=\left.\operatorname{Res}_{y}\left(f(x, y)-u_{0}, g(x, y)-v_{0}\right)\right|_{x=x_{0}}=A\left(x_{0}, u_{0}, v_{0}\right)$

But the latter contradicts the hypothesis that $A\left(x_{0}, u_{0}, v_{0}\right)=A_{0}\left(u_{0}, v_{0}\right) \neq 0$.

(ii) $\Rightarrow$ (iii). Using Property 1 , we get that $B(y, f, g)=0$. Since $r=0, B(y, u, v)=$ $B_{0}(u, v)$. Hence $B_{0}(f, g)=0$. But $B_{0}(u, v) \neq 0$.

(iii) $\Leftrightarrow$ (iv). Let $\varphi(u, v)$ be of minimal positive degree so that $\varphi(f, g)=0$. Then

$$
\left[\begin{array}{ll}
\frac{\partial f}{\partial x} & \frac{\partial g}{\partial x} \\
\frac{\partial f}{\partial y} & \frac{\partial g}{\partial y}
\end{array}\right]\left[\begin{array}{l}
\frac{\partial \varphi}{\partial u}(f, g) \\
\frac{\partial \varphi}{\partial v}(f, g)
\end{array}\right]=\left[\begin{array}{l}
0 \\
0
\end{array}\right]
$$

By minimality, we note that either $\frac{\partial \varphi}{\partial u}(f, g) \neq 0$ or $\frac{\partial \varphi}{\partial v}(f, g) \neq 0$. Thus $J(f, g)=0$.

Conversely, assume that $f, g$ are algebraically independent. Then since $A(x, f, g)=$ $B(y, f, g)=0$, we see that there exist polynomials $K(x, u, v)$ and $H(y, u, v)$ of minimal positive degrees in $x, y$ respectively, so that $K(x, f, g)=H(y, f, g)=0$. Then

$$
\left[\begin{array}{ll}
\frac{\partial K}{\partial u}(x, f, g) & \frac{\partial K}{\partial v}(x, f, g) \\
\frac{\partial H}{\partial u}(y, f, g) & \frac{\partial H}{\partial v}(y, f, g)
\end{array}\right]\left[\begin{array}{ll}
\frac{\partial f}{\partial x} & \frac{\partial f}{\partial y} \\
\frac{\partial g}{\partial x} & \frac{\partial g}{\partial y}
\end{array}\right]=\left[\begin{array}{cc}
-\frac{\partial K}{\partial x}(x, f, g) & 0 \\
0 & -\frac{\partial H}{\partial y}(y, f, g)
\end{array}\right] \text {. }
$$

But $(\partial K) /(\partial x)(x, f, g) \cdot(\partial H) /(\partial y)(y, f, g) \neq 0$, and thus $J(f, g) \neq 0$.

(iv) $\Rightarrow$ (i). Assume that $k \geqslant 1$. Pick $\left(u_{0}, v_{0}\right) \in \mathbb{C}^{2}$ so that $A_{k}\left(u_{0}, v_{0}\right) A_{0}\left(u_{0}, v_{0}\right) \neq 0$ and let $x_{0} \in \mathbb{C}$ be such that $A\left(x_{0}, u_{0}, v_{0}\right)=0$. By a property similar to Property 3 , we can find $y_{0} \in \mathbb{C}$ such that $f\left(x_{0}, y_{0}\right)-u_{0}=g\left(x_{0}, y_{0}\right)-v_{0}=0$. Furthermore, we note that the polynomials $f(x, y)-u_{0}$ and $g(x, y)-v_{0}$ have no common factor of positive degree for otherwise the common factor $h(x, y)$ has positive $y$-degree and $A\left(x, u_{0}, v_{0}\right)=0$ by Property 5 , contradicting $A_{0}\left(u_{0}, v_{0}\right) \neq 0$. Let $\bar{f}(x, y)=f\left(x+x_{0}, y+y_{0}\right)-u_{0}$, $\bar{g}(x, y)=g\left(x+x_{0}, y+y_{0}\right)-v_{0}$. Then $\bar{f}(0,0)=\bar{g}(0,0)=0$ and $J(\bar{f}, \bar{g})=0$. Using (iii) we can find $\varphi(u, v)$ of minimal positive degree so that $\varphi(\bar{f}, \bar{g})=0$. Furthermore, since $\varphi(0,0)=0, \varphi(u, v)$ has no constant term. In that case we see that $\bar{f}(x, y)$ and $\bar{g}(x, y)$ have a common factor of positive degree, $d(x, y)$ say. The latter implies that $d\left(x-x_{0}, y-y_{0}\right)$ is a common factor of positive degree of $f(x, y)-u_{0}$ and $g(x, y)-v_{0}$. 
REMARK 1. Quasi-regularity cannot be dropped from the hypothesis of the theorem as the following example indicates: Let $f(x, y)=x y+1, g(x, y)=x y+2$. Then $J(f, g)=$ 0 but $A(x, u, v)=x(u-v+1), B(y, u, v)=y(u-v+1)$ and thus $k=r=1$.

The above theorem takes a special form when $f(x, y)$ and $g(x, y)$ are homogeneous polynomials. Before we can state it we shall need the following result, due to Swan, which is an easy consequence of Property 4.

Lemma 1. Let $n, m \geqslant 1, a, b \in \mathbb{C}, a b \neq 0$. Then

$$
\operatorname{Res}_{x}\left(a x^{n}-u, b x^{m}-v\right)=(-1)^{n}\left(a^{m / d} v^{n / d}-b^{n / d} u^{m / d}\right)^{d},
$$

where $d=\operatorname{gcd}(m, n)$.

In view of the above, we then have the following well-known result:

COROLLARY 1. Let $f(x, y), g(x, y)$ be homogeneous polynomials, not necessarily quasi-regular in $x, y$, of positive degrees $n, m$ respectively. Then

$$
J(f, g)=0 \Leftrightarrow c f^{m / d}=g^{n / d},
$$

where $c \in \mathbb{C}, d=\operatorname{gcd}(m, n)$. In particular, $c f=g$ if $m=n$.

Proof: By a linear change of coordinates we may assume that $f(x, y)$ and $g(x, y)$ are quasi-regular in $x$. Suppose first that $J(f, g)=0$. Then $\operatorname{Res}_{x}(f-u, g-v)=$ $B(y, u, v)=B_{0}(y, u, v)=\operatorname{Res}_{x}\left(a x^{n}-u, b x^{m}-v\right)=(-1)^{n}\left(a^{m / d} v^{n / d}-b^{n / d} u^{m / d}\right)^{d}$, where $a, b$ are the coefficients of $x^{n}, x^{m}$ in $f(x, y), g(x, y)$ respectively. But $B_{0}(f, g)=$ 0 . The converse is trivial.

CoROLlary 2. Let $f(x, y), g(x, y)$ be polynomials of positive degrees in $x$, quasi-regular in $x$. Then

$$
J(f, g)=0 \Rightarrow \operatorname{Res}_{x}(f, g)=c, c \text { is a constant. }
$$

Proof: $\operatorname{Res}_{x}(f, g)=B_{0}(0,0)$.

Quasi-regularity is essential in the hypothesis of the above corollary as Remark 1 shows. Also, as a consequence of the above corollary and Theorem 1 , if $f, g$ are algebraically dependent polynomials, then they either have no zeros or they have a common factor of positive degree.

When only one parameter is allowed in (1), Theorem 1 takes a somewhat different form. We begin with the following. Let $h(x, y)$ be irreducible in $\mathbb{C}[x, y]$ of positive degree in both $x$ and $y$, quasi-regular in $x$ and $y$. Consider

$$
p(y, u)=\operatorname{Res}_{x}(f-u, h)=\sum_{j=0}^{\lambda} p_{j}(u) y^{j}
$$


where $\lambda$ is the $y$-degree of $p(y, u)$. Note that $p_{0}(u)$ has positive degree because $h$ has positive degree in $x$. The following theorem provides a necessary and sufficient condition for $\lambda=0$.

THEOREM 2. Let $h, p(y, u)$ be as above. Then the following conditions are equivalent:

(i) $\lambda=0$.

(ii) $h(x, y)$ divides $J(f, h)$.

(iii) There is a unique $u_{0}$ so that $h$ divides $f-u_{0}$.

(iv) $p_{0}(u)=c\left(u-u_{0}\right)^{q}$, where $q=\operatorname{deg}_{x} h(x, y), c \in \mathbb{C}$.

Proof: (i) $\Rightarrow$ (ii). Let $u_{0}$ be such that $p_{0}\left(u_{0}\right)=0$. Then $f-u_{0}=h \cdot d$ by Property 2 and a computation shows that $J(f, h)=h \cdot J(d, h)$.

(ii) $\Rightarrow$ (iii). For this we consider the following cases:

a) $\quad h$ divides $f_{y}$. We are going to show that $\lambda=0$. We argue by contradiction. Suppose then that $\lambda \geqslant 1$. Pick $u_{0} \in \mathbb{C}$ so that $p_{\lambda}\left(u_{0}\right) \neq 0$ and let $y_{0} \in \mathbb{C}$ be such that $p\left(y_{0}, u_{0}\right)=0$. By Property 3 , we can find $x_{0} \in \mathbb{C}$ with the property that $f\left(x_{0}, y_{0}\right)-u_{0}=h\left(x_{0}, y_{0}\right)=0$. We also note that $h$ divides $f_{x}$ since it divides $J(f, h)$. Therefore $f\left(x_{0}, y_{0}\right)-u_{0}=f_{x}\left(x_{0}, y_{0}\right)=f_{y}\left(x_{0}, y_{0}\right)=0$ and $\left\{(x, y) \in \mathbb{C}^{2} \mid f-u_{0}=0\right\}$ is a singular curve (over $\mathbb{C}^{2}$ ). Now let $S=\left\{u \in \mathbb{C} \mid p_{\lambda}(u) \neq 0\right\}$, and for each $u \in S$, let $C_{u}$ be the curve $\left\{(x, y) \in \mathbb{C}^{2} \mid f-u=0\right\}$. We observe that every $C_{u}$ is singular (over $\mathbb{C}^{2}$ ). But $C_{v}$ is singular if and only if $v \in f\left[(x, y) \mid f_{x}=f_{y}=0\right]$, an impossibility as Sard's theorem indicates, [5]. Thus $\lambda=0$.

$\beta) h$ does not divide $f_{y}$. Let $\left(x_{0}, y_{0}\right) \in \mathbb{C}^{2}$ be with the properties $h\left(x_{0}, y_{0}\right)=0$ and $f_{y}\left(x_{0}, y_{0}\right) \cdot h_{y}\left(x_{0}, y_{0}\right) \neq 0$, and let $u_{0}=f\left(x_{0}, y_{0}\right)$. In that case using the Inverse Function theorem we can find $C^{\infty}$ functions $y=\varphi(x), y=\psi(x)$ with $\varphi\left(x_{0}\right)=\psi\left(x_{0}\right)=y_{0}$ and $h(x, \varphi(x))=f(x, \psi(x))-u_{0}=0$ in a neighbourhood $U$ of $x_{0}$. But since $h$ divides $J(f, h)$ we conclude that $\varphi^{\prime}(x)=\psi^{\prime}(x)$ near $x_{0}$. That implies $\varphi(x)=\psi(x)$ in $U$, and thus $h$ divides $f-u_{0}$. Finally, we note that $u_{0}$ is unique since $h$ is irreducible.

(iii) $\Rightarrow$ (iv) Let $u_{0}, u_{1}$ be zeros of $p_{0}(u)$. Then $f-u_{0}=h \cdot d_{0}, f-u_{1}=h \cdot d_{1}$, and thus $u_{1}-u_{0}=h\left(d_{0}-d_{1}\right)$ or $u_{1}=u_{0}$. Therefore $p_{0}(u)=c\left(u-u_{0}\right)^{k}$, for some $c \in \mathbb{C}$, $k \geqslant 1$. But then since $h$ is quasi-regular in $x, h(x, 0)$ has $q$ zeros-counted with multiplicities. That, along with Property 4 , shows that $k=q$.

Now let $g=h_{1}^{n_{1}} h_{2}^{n_{2}} \cdots h_{s}^{n_{s}}, n_{j} \geqslant 1$, be the prime factorisation of $g$ in $\mathbb{C}[x, y]$, and let $q_{i}=\operatorname{deg} h_{i}(x, y), i=1, \ldots, s$. Note that every $h_{i}(x, y)$ is quasi-regular in $x$ and $y$.

Using the previous theorem and Property 5 we can obtain the following:

Corollary 3. Let $f, g, h_{i}$ be as above. Then

$$
\operatorname{Res}_{x}(f-u, g)=p(u) \Leftrightarrow h_{i} \text { divides } J\left(f, h_{i}\right) \text { for all } i=1, \ldots, s .
$$


(ii) Suppose that $\operatorname{Res}_{x}(f-u, g)=p(u)$. Then

$$
p(u)=c \prod_{i=1}^{\dot{0}}\left(u-u_{i}\right)^{m_{i}}, u_{i}, c \in \mathbb{C} \text { and } m_{i}=n_{i} \cdot q_{i}, i=1, \ldots, s .
$$

\section{The InVertibility of $F$}

Let $f(x, y), g(x, y)$ be as before and let $n_{1}=\operatorname{deg} f(x, 0), n_{2}=\operatorname{deg} f(0, y), m_{1}=$ $\operatorname{deg} g(x, 0), m_{2}=\operatorname{deg} g(0, y)$ and $F=(f, g): \mathbb{C}^{2} \rightarrow \mathbb{C}^{2}$. For convenience we assume that $F(0,0)=(0,0)$. In this section we shall state a necessary and sufficient condition, in terms of the polynomials $A(x, u, v)$ and $B(y, u, v)$, for the map $F$ to be $1-1$ and onto. Our results are similar to the ones in $[1]$, and they come as a natural by-product of our earlier considerations.

Lemma 2. Let $f, g, F, A(x, u, v)$ and $B(y, u, v)$ be as above. Then $F$ is $1-1$ and onto $\Rightarrow A(x, u, v)=a x+A_{0}(u, v), B(y, u, v)=b y+B_{0}(u, v)$, where $a, b \in \mathbb{C}, a b \neq 0$.

Proof: We shall first prove that $k=r=1$. We first note by Property 1 that $k, r \geqslant 1$ since $F$ is onto. Pick $\left(u_{0}, v_{0}\right) \in \mathbb{C}^{2}$ so that $A_{k}\left(u_{0}, v_{0}\right) \neq 0$. Since $F$ is an automorphism, $f$ and $g$ are quasi-regular in $x[3]$, and thus by Property 3 the polynomial $p(x)=A\left(x, u_{0}, v_{0}\right)$ has only one root, say $x_{0}$. We are going to compute $p^{\prime}\left(x_{0}\right)$. Let the unique $y_{0}$ be such that $f\left(x_{0}, y_{0}\right)-u_{0}=g\left(x_{0}, y_{0}\right)-v_{0}=0$. Now let $\bar{x}=x, \bar{y}=y-y_{0}$ and write $f(x, y)=\bar{f}(\bar{x}, \bar{y}), g(x, y)=\bar{g}(\bar{x}, \bar{y})$. Observe that $\operatorname{deg} f\left(x_{0}, y\right)=\operatorname{deg} \bar{f}\left(x_{0}, \bar{y}\right)$ and $\operatorname{deg} g\left(x_{0}, y\right)=\operatorname{deg} \bar{g}\left(x_{0}, \bar{y}\right)$. Now using the Chain rule for resultants $[4$, Theorem 6, p.349] we have :

$$
\operatorname{Res}_{y}\left(f(x, y)-u_{0}, g(x, y)-v_{0}\right)=\operatorname{Res}_{\bar{y}}\left(f(\bar{x}, \bar{y})-u_{0}, \bar{g}(\bar{x}, \bar{y})-v_{0}\right) .
$$

We get $p^{\prime}\left(x_{0}\right)$ by differentiating the above resultant with respect to $\bar{x}$ and evaluating the result at $\bar{x}=x_{0}$. This amounts to differentiating the last column only of the determinant defining the resultant, since $\bar{f}\left(x_{0}, 0\right)-u_{0}=\bar{g}\left(x_{0}, 0\right)-u_{0}=0$. By expanding the resulting determinant by its last column and then expanding the two cofactors by their last columns, we get:

$$
p^{\prime}\left(x_{0}\right)=(-1)^{n_{2}}\left[\operatorname{Res}_{\bar{y}}\left(\frac{\bar{f}\left(x_{0}, \bar{y}\right)-u_{0}}{\bar{y}}, \frac{\bar{g}\left(x_{0}, \bar{y}\right)-v_{0}}{\bar{y}}\right)\right] \cdot J(\bar{f}, \bar{g})\left(x_{0}, 0\right)
$$

The above shows that $p^{\prime}\left(x_{0}\right) \neq 0$, since 0 is the only common root of $\bar{f}\left(x_{0}, \bar{y}\right)-u_{0}$ and $\bar{g}\left(x_{0}, \bar{y}\right)-v_{0}$, and thus $k=1$. Similarly, we can prove that $r=1$. Now suppose that $A_{1}(u, v)$ is not a nonzero constant. In that case we pick $\left(u_{1}, v_{1}\right) \in \mathbb{C}^{2}$ so that $A_{1}\left(u_{1}, v_{1}\right)=0$. Then, depending upon whether $A_{0}\left(u_{1}, v_{1}\right)$ is nonzero or zero, the 
polynomials $f-u_{1}$ and $g-v_{1}$ will either have no common zero or will have a common factor of positive degree. But this is a contradiction to $F$ being $1-1$ and onto.

We say that $F$ has a polynomial inverse if there is a polynomial map $G(x, y)=$ $(p(x, y), q(x, y))$ so that $G \circ F(x, y)=(x, y)$. For a polynomial map $F=(f, g)$ we define its degree, $\operatorname{deg} F(x, y)$, to be the highest degree of the monomials in $f(x, y)$ and $g(x, y)$.

The following Proposition describes precisely what the inverse $G$ of $F$ is, in the case where $F$ is $1-1$ and onto.

Proposition 1. Let $F, a, b, A_{0}(u, v), B_{0}(u, v)$ be as in Lemma 2 and $G(x, y)=\left(-\left(A_{0}(x, y)\right) / a,-\left(B_{0}(x, y)\right) / b\right)$. Then $G$ is the inverse of $F(x, y)$. Furthermore, $\operatorname{deg} F(x, y)=\operatorname{deg} G(x, y)$.

Proof: In view of Lemma 2 and Property 1 we have:

$$
G \circ F(x, y)=G(f, g)=\left(-\frac{A_{0}(f, g)}{a},-\frac{B_{0}(f, g)}{b}\right)=(x, y) \text {. }
$$

For the second assertion, we note that $A_{0}(u, v)=\operatorname{Res}_{y}(f(0, y)-u, g(0, y)-v)$, and thus $\operatorname{deg} A_{0}(u, v)=\max \left(n_{2}, m_{2}\right)$ and, similarly $\operatorname{deg} B_{0}(u, v)=\max \left(n_{1}, m_{1}\right)$. Thus, $\operatorname{deg} G=\max \left(n_{1}, n_{2}, m_{1}, m_{2}\right)$. But since the Newton polygons of an automorphism are triangles we see that $\operatorname{deg} F(x, y)=\operatorname{deg} G(x, y),[3]$.

Finally, we may use the so-called "border polynomials" of $F(x, y)$ to describe explicitly its inverse, $G(x, y)$. These are $f(x, 0), g(x, 0), f(0, y)$ and $g(0, y)$. Using Lemma 2 we get:

$$
A_{0}(u, v)=\operatorname{Res}_{y}(f(0, y)-u, g(0, y)-v), B_{0}(u, v)=\operatorname{Res}_{x}(f(x, 0)-u, g(x, 0)-v)
$$

Furthermore, (3) together with the Chain rule for resultants shows that

$$
a=(-1)^{n_{2}}\left[\operatorname{Res}_{y}\left(\frac{f(0, y)}{y}, \frac{g(0, y)}{y}\right)\right] \cdot J(f, g)(0,0) .
$$

Likewise

$$
b=(-1)^{n_{1}}\left[\operatorname{Res}_{x}\left(\frac{f(x, 0)}{x}, \frac{g(x, 0)}{x}\right)\right] \cdot J(f, g)(0,0)
$$

Thus,

Proposition 2. If $F=(f, g)$ is $1-1$ and onto, $F$ has a polynomial inverse $G$ which is completely determined by the border polynomials of $F$. 


\section{A Conjecture}

Let $F=(f, g)$ be as before, but not necessarily $F(0,0)=(0,0)$. It is clear that $F$ has no zeros if there exist polynomials $\varphi(x, y), \psi(x, y)$ so that $f \varphi+g \psi=1$. The latter is equivalent-by Property 3-to the fact that $\operatorname{Res}_{x}(f, g)=c_{1}$ and $\operatorname{Res}_{y}(f, g)=c_{2}$, $c_{1} c_{2} \neq 0, c_{i} \in \mathbb{C}$.

In Section 3 we saw that if $J(f, g)=0$, then $\operatorname{Res}_{x}(f, g)=c, c \in \mathbb{C}$. Furthermore, (Corollary 3), whenever every irreducible factor $h$ of $g$ divides $J(f, h)$, then $\operatorname{Res}_{x}(f, g)=c$. We believe that a partial converse of Corollary 2 is true; we state it in the form of the following:

ConJECTURE. Let $f(x, y), g(x, y)$ be quasi-regular in $x$. Then $\operatorname{Res}_{x}(f, g)=c$, $c \in \mathbb{C} \Rightarrow$ there exists a point $\left(x_{0}, y_{0}\right) \in \mathbb{C}^{2}$ for which $J(f, g)\left(x_{0}, y_{0}\right)=0$.

REMARK 2. We note that the above conjecture is trivially true in the case where $c=0$.

We conclude with a consequence of the above conjecture in relation to the Jacobian conjecture.

Remark 3. Let $F=(f, g): \mathbb{C}^{2} \rightarrow \mathbb{C}^{2}$ be such that $J(f, g)=1$. Then, if the above conjecture is true, $F$ is onto.

Proof: By a linear change of coordinates we may suppose that $f, g$ are quasiregular in $x$. Now, if there exists a point $(s, t)$ for which $F^{-1}(s, t)=\emptyset$, then $\operatorname{Res}_{x}(f-s, g-t)=c \neq 0$. But $J(f-s, g-t)=J(f, g)=1$, contradicting the conjecture.

\section{REFERENCES}

[1] K. Adjamagbo and A. van den Essen, 'A resultant criterion and formula for the inversion of a polynomial map in two variables', J. Pure Appl. Algebra 64 (1990), 1-6.

[2] G.E. Collins, 'The calculation of multivariate polynomial resultants', J. Assoc. Comput. Mach. 18 (1971), 515-532.

[3] J.H. McKay and S.S-S. Wang, 'An inversion formula for two polynomials in two variables', J. Pure Appl. Algebra 40 (1986), 245-257.

[4] J. H. McKay and S.S-S. Wang, 'A chain rule for the resultant of two polynomials', Arch. Math. 53 (1989), 347-351.

[5] A. Sard, 'The measure of the critical values of differentiable maps', Bull. Amer. Math. Soc. 48 (1942), 883-897.

[6] B.L. van der Waerden, Algebra Vol. 1 (New York, 1970).

Department of Mathematical Sciences

Oakland University

Rochester MI 48309

United States of America 